\title{
Facile Fabrication of $\mathrm{SrTiO}_{3} @ \mathrm{MoS}_{2}$ Composite Nanofibers for Excellent Photodetector Application
}

\author{
Jinghong Li, ${ }^{1}$ Lun Zhang, ${ }^{1}$ Di Wang, ${ }^{2}$ Ran Wang, ${ }^{1}$ Xiujuan Liu, ${ }^{3}$ and Jingxin Zhou $\mathbb{D}^{1}$ \\ ${ }^{1}$ Hebei Key Laboratory of Applied Chemistry, \\ Heavy Metal Deep-Remediation in Water and Resource Reuse Key Lab of Hebei Province, \\ School of Environmental and Chemical Engineering, Yanshan University, Qinhuangdao 066004, China \\ ${ }^{2}$ School of Materials Science and Engineering, Shijiazhuang Tiedao University, Shijiazhuang 050043, China \\ ${ }^{3}$ The First Hospital of Qinhuangdao City, Qinhuangdao 066000, China
}

Correspondence should be addressed to Jingxin Zhou; zhoujingxin@ysu.edu.cn

Received 31 May 2020; Accepted 29 June 2020; Published 23 July 2020

Academic Editor: Peizhi Guo

Copyright (c) 2020 Jinghong Li et al. This is an open access article distributed under the Creative Commons Attribution License, which permits unrestricted use, distribution, and reproduction in any medium, provided the original work is properly cited.

Molybdenum disulfide $\left(\mathrm{MoS}_{2}\right)$, as a kind of transition metal dichalcogenide, has been widely studied for its excellent compatibility with most of inorganic nanomaterials. Nevertheless, its microscale and agglomeration limit the performance severely. Therefore, the special structure of $\mathrm{V}-\mathrm{MoS}_{2}$ has drawn a lot of interest, which can not only reduce the size of $\mathrm{MoS}_{2}$ nanosheets but also improve the valence electron structure of the materials. In this work, $\mathrm{SrTiO}_{3} @ \mathrm{MoS}_{2}$ composite nanofibers were synthesized by the simple electrospinning and hydrothermal method, and it was applied as a novel material for photodetector. SEM, TEM, EDX, XRD, I-T curves, and EIS analysis were used to study the structure and properties of the prepared $\mathrm{SrTiO}_{3} @ \mathrm{MoS}_{2} \mathrm{composite}$ nanofibers. Simulating under sunlight at a potential of $1.23 \mathrm{~V}$, the prepared composite materials exhibited a superior photoelectric performance of photocurrent density of $21.4 \mu \mathrm{A}$ and a resistance of $2.3 \Omega$. These results indicate that the composite of $\mathrm{SrTiO}_{3}$ nanofiber adhered with $\mathrm{V}-\mathrm{MoS}_{2}$ has a stable composite structure, good electrical conductivity, and photoelectric sensitivity and is a suitable material for photodetectors. This work provides new ideas for the preparation of self-assembled materials and their application in photodetectors.

\section{Introduction}

As we all know, water pollution, greenhouse effect, and fumes are hazardous to human beings, but few people notice that potential light pollution is also threatening our health [1-4]. Light is everywhere in our life; in fact, proper sunlight is useful for our health. However, excessive light may damage our eyesight, affect our emotions, and even induce cancer [5]. The light pollution mainly originated from assimilation lighting and massive use of architectural glass, particularly in the developed region. To solve this problem, photodetector has been widely studied as optoelectronic devices [6-8].

As a member of semiconductors, transition metal dichalcogenides (TMDCs) have shown perfect properties of its special band structure, fairly physical character, and superconductive performance $[9,10]$. Within all kinds of materials of TMDCs, molybdenum disulfide $\left(\mathrm{MoS}_{2}\right)$ has attracted more and more attention due to its special structure, excellent compatibility with abundant nanoscale materials, and attractive price [11]. Therefore, $\mathrm{MoS}_{2}$ has been used in many fields such as nano-electronic devices and optoelectronics. However, the performance of $\mathrm{MoS}_{2}$ was limited by the lamellae stacking and the lack of active sites [12]. Recently, vertical $\mathrm{MoS}_{2}\left(\mathrm{~V}-\mathrm{MoS}_{2}\right)$ has been reported frequently in the field of photoelectricity [13]. The photoelectric performance of $\mathrm{MoS}_{2}$ nanosheets has been improved by building a $\mathrm{V}-\mathrm{MoS}_{2}$ structure at the nanoscale and changing $\mathrm{MoS}_{2}$ from a multilayer structure to a single-layer structure. Moreover, high-quality $\mathrm{V}-\mathrm{MoS}_{2}$ structure has a stable reproducible optoelectronic performance and is easy to fabricate. In addition, $\mathrm{V}-\mathrm{MoS}_{2}$ with a single-layer 
structure can form Mo-O bonds, which can further improve the sensitivity of the photodetector [14-16]. However, many efforts have been made to construct the $\mathrm{V}-\mathrm{MoS}_{2}$ structure, but most of them are still unsatisfactory for their poor performance and instability.

In this work, porous $\mathrm{SrTiO}_{3}$ nanofibers embedded with $\mathrm{MoS}_{2}$ nanosheets were constructed through electrospinning and hydrothermal method, which showed a $\mathrm{V}-\mathrm{MoS}_{2}$ structure [17-20]. The porous nanofibers can provide a stable substrate for delamination of $\mathrm{MoS}_{2}$ to form a vertical monolayer structure. The prepared $\mathrm{MoS}_{2}$-based material exhibited a sensitive optoelectronic performance through current density curves and Nyquist EIS plots characterization [21-23].

\section{Materials and Methods}

2.1. Materials. PVP (molecular weight $=1300$ 000), thioacetamide $\left(\mathrm{C}_{2} \mathrm{H}_{5} \mathrm{NS}\right)$, tetrabutyl titanate (TBOT; $\left.\mathrm{C}_{16} \mathrm{H}_{36} \mathrm{O}_{4} \mathrm{Ti}\right)$, acetic acid $\left(\mathrm{C}_{2} \mathrm{H}_{4} \mathrm{O}_{2}\right)$, 2-methoxyethanol $\left(\mathrm{C}_{3} \mathrm{H}_{8} \mathrm{O}_{2}\right)$, strontium acetate $\left(\mathrm{C}_{4} \mathrm{H}_{6} \mathrm{O}_{4} \mathrm{Sr}\right)$, sodium molybdate $\left(\mathrm{Na}_{2} \mathrm{MoH}_{4} \mathrm{O}_{6}\right)$, thioacetamide $\left(\mathrm{C}_{2} \mathrm{H}_{5} \mathrm{NS}\right)$, Pluronic F127, and hexadecyl trimethylammonium bromide (CTAB) were purchased from Aladdin Reagent Co, Ltd. All chemical reagents were of analytical grade (AR) and used without any further purification. The ultrapure water used throughout the experiments was extracted from a Milli-Q Millipore All Filter system (Millipore Co, Bedford, MA, USA).

2.2. Synthesis of Porous SrTiO3 Nanofibers. In brief, tetrabutyl titanate (TBOT) and strontium acetate were dissolved in $10 \mathrm{~mL}$ acetic acid at a mass ratio of $1.6: 1$. The mixture was vigorous stirred for 15 min until the solution transformed to transparent, and it was named solution A. Then, PVP, Pluronic F127, and CTAB were put into $6 \mathrm{~mL} 2$-methoxyethanol at a mass ratio of $1: 0.5: 0.7$ with vigorous stirring for $15 \mathrm{~min}$, which has been deeply explored in previous work to ensure the proper ratio of these reagents [17]. When the mixture became transparent viscous liquid, solution A was dropped to the liquid slowly and stirred overnight. Next, the mixture was put into syringe and applied a $15 \mathrm{kV}$ electric voltage at the distance of $20 \mathrm{~cm}$. The ejected rate was set $0.5 \mathrm{ml} \mathrm{h}^{-1}$ to obtain the nanofiber membrane. Then, the prepared membrane was under pyroprocessing at $700^{\circ} \mathrm{C}$ for $3 \mathrm{~h}$ at a ramp rate of $2^{\circ} \mathrm{C} \min ^{-1}$ to obtain the porous $\mathrm{SrTiO}_{3}$ nanofibers.

2.3. Synthesis of SrTiO3@MoS2 2D Structure. Typically, $\mathrm{C}_{2} \mathrm{H}_{5} \mathrm{NS}$ and $\mathrm{Na}_{2} \mathrm{MoO}_{4} \cdot 2 \mathrm{H}_{2} \mathrm{O}$ with a mass ratio of $3: 5$ were dissolved in $15 \mathrm{~mL}$ ultrapure water. Then, $150 \mathrm{mg}$ porous $\mathrm{SrTiO}_{3}$ nanofibers were put into the solution. The mixed solution was then shifted to a $50 \mathrm{~mL}$ hydrothermal reactor and heated at $220^{\circ} \mathrm{C}$ for $24 \mathrm{~h}$. Ultimately, the $\mathrm{SrTiO}_{3} @ \mathrm{MoS}_{2}$ $2 \mathrm{D}$ structure was obtained through filtration separation and vacuum drying [24].

2.4. Photo(electro)chemical Measurements.8 mg $\mathrm{SrTiO}_{3} @$ $\mathrm{MoS}_{2}$ sample and $1 \mathrm{mg}$ acetylene black were transferred to a
$25 \mathrm{~mL}$ beaker with $10 \mathrm{~mL}$ absolute alcohol, and then, $10 \mu \mathrm{L}$ PTFE was dropped into the mixture with vigorous stirring. The mixed solution was ultrasonic treated for about $30 \mathrm{~min}$ and shaken well at last $2 \mathrm{~min}$. After that, the beaker was put into a vacuum drying oven to evaporate the alcohol for $3 \mathrm{~h}$ and then transferred the sediment to the nickel foam. Finally, put the nickel foam into a vacuum oven at $100^{\circ} \mathrm{C}$ overnight and obtain the working electrodes. The photo (electro) chemical test was performed using a CHI660 electrochemical workstation with a standard three-electrode system with a graphene rod, an $\mathrm{Ag} / \mathrm{AgCl}$ electrode, and nickel foam, respectively. All the measurements were tested in $1 \mathrm{M} \mathrm{KOH}$ aqueous solution under simulated solar light, which was provided by a $300 \mathrm{~W}$ Xe lamp. The change in the current density curve with time was tested at a potential of $1.23 \mathrm{~V}$ under light on/off condition [25]. The electrochemical impedance spectroscopy (EIS) tests were measured in a frequency range from $100 \mathrm{kHz}$ to $0.1 \mathrm{~Hz}$ with an amplitude of $5 \mathrm{mV}$ at $\eta=-0.24 \mathrm{~V}[26-30]$.

2.5. Characterizations. X-ray diffraction (XRD) analyses of the as-prepared samples were investigated with an X-ray diffractometer (SmartLab, Rigaku, Akishima, Japan). The morphologies of prepared samples were obtained by using transmission electron microscopy (TEM) (HT7700, Hitachi High Technologies Corporation, Ibaraki, Japan) and fieldemission scanning electron microscopy (SEM, S-4800II, Hitachi, Japan). Elemental map results were investigated with an Oxford Link-ISIS X-ray EDXS microanalyzer at $200 \mathrm{kV}$ with SEM. All the ultrapure water used in the experiment was purified in a Milli-Q Millipore All Filter system (Millipore Co, Bedford, MA, USA).

\section{Results and Discussion}

3.1. Characterization of SrTiO3@MoS . The morphologies of as-prepared materials were obtained by transmission electron microscopy (TEM) and scanning electron microscopy (SEM). Figure 1(a) shows the SEM image of electrospun nanofibers before calcined, which mainly consisted of PVP, F127, and other ions. The diameter of nanofibers is approximately $1-3 \mu \mathrm{m}$ and evenly distributed with a network structure. Figure 1(b) shows the image of nanofibers after being calcined at $700^{\circ} \mathrm{C}$, and the surface of fibers becomes rough and shows many pores. It can be clearly observed that the diameter of fiber reduced to about $300 \mathrm{~nm}$ with $10-20 \mathrm{~nm}$ aperture gap uniform distributes on the surface. This special morphology is mainly caused by the synergistic effect of F127 and CTAB, which has been studied systematically in the earlier works. This special structure can greatly increase the surface area of $\mathrm{SrTiO}_{3}$ NFs and provide suitable sites for loading $\mathrm{MoS}_{2}$ [31-36]. In addition, the pores in the nanofibers can magnify the contact area between electrodes and electrolytes and optimize the structure of valence electron transmission. Figure 1(c) exhibits the result of $\mathrm{SrTiO}_{3} @ \mathrm{MoS}_{2}$ composite nanofibers. It can be found that the triangular $\mathrm{MoS}_{2}$ nanosheets firmly embed in the aperture gap of $\mathrm{SrTiO}_{3}$ nanofibers to form a V-MoS 2 structure. TEM is carried out to further 


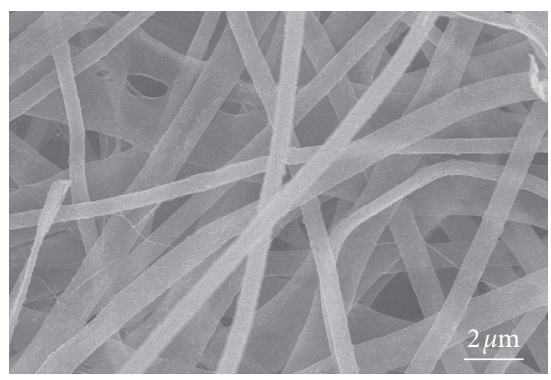

(a)

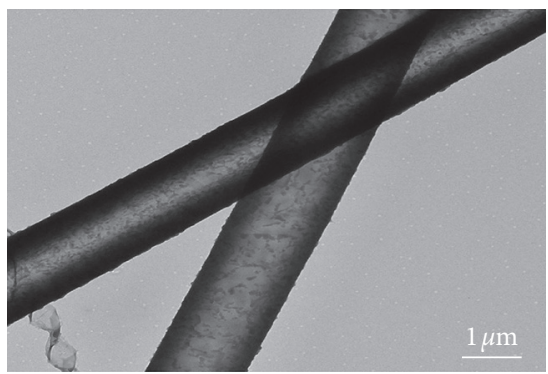

(d)

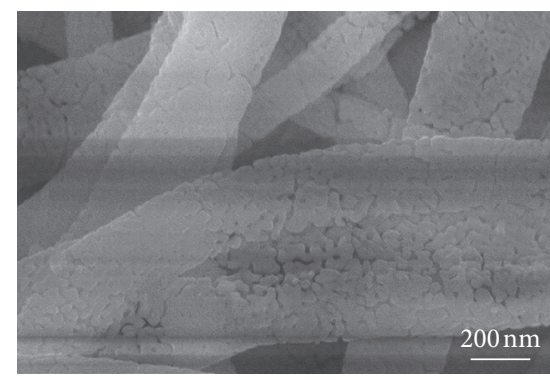

(b)

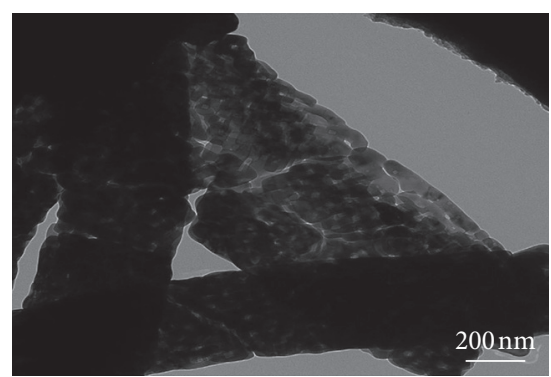

(e)

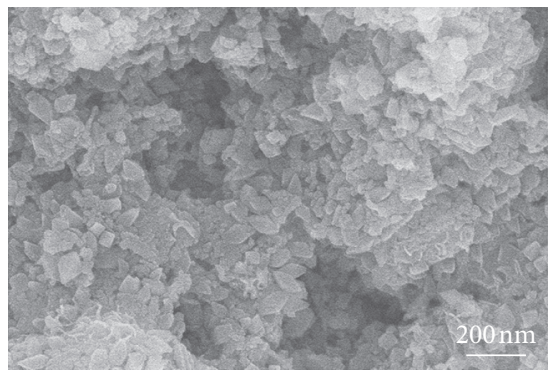

(c)

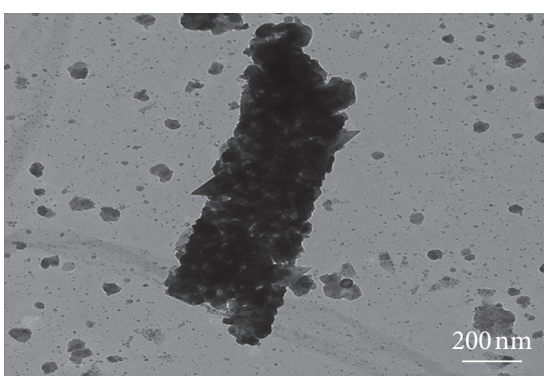

(f)

FIgURE 1: SEM images of (a) nanofibers before calcination, (b) porous $\mathrm{SrTiO}_{3} \mathrm{NFs}$, and (c) $\mathrm{SrTiO}_{3} @ \mathrm{MoS}_{2}$ composite nanofibers. TEM images of (d) nanofibers before calcination, (e) porous $\mathrm{SrTiO}_{3} \mathrm{NFs}$, and (f) $\mathrm{SrTiO}_{3} @ \mathrm{MoS}_{2}$ composite nanofibers.

explore the structure of $\mathrm{MoS}_{2}$ on $\mathrm{SrTiO}_{3} @ \mathrm{MoS}_{2}$ hybrids, as shown in Figures 1(d)-1(f). From Figure 1(d), the diameter of as-spun nanofibers is about $200 \mathrm{~nm}$, which corresponds to the SEM images. As shown in Figure 1(e), it can be observed that the calcined nanofibers are highly porous due to the different electron penetrability of various positions [37-46]. Figure 1(f) shows the composite nanofibers of $\mathrm{SrTiO}_{3} @ \mathrm{MoS}_{2}$, and it can be clearly seen that the $\mathrm{MoS}_{2}$ nanosheets and porous nanofibers combine tightly. Furthermore, the sample was under ultrasonic treatment for about $15 \mathrm{~min}$ before TEM operation, and the $\mathrm{MoS}_{2}$ nanosheets were still adhered in the nanofibers. This phenomenon can convincingly demonstrate the stability of $\mathrm{SrTiO}_{3} @ \mathrm{MoS}_{2}$ composite nanomaterials. The mesoporous structure in NFs provided an excellent adhesion environment to form the $\mathrm{V}-\mathrm{MoS}_{2}$ structure [47].

The elemental map is one of the most commonly used techniques to observe the morphology of elements distribution. The SEM mapping of bare $\mathrm{SrTiO}_{3}$ nanofibers and $\mathrm{SrTiO}_{3} @$ $\mathrm{MoS}_{2}$ hybrids is shown in Figure 2. Figures 2(a)-2(d) illustrate that the elemental map of $\mathrm{SrTiO}_{3}$ nanofibers includes $\mathrm{Ti}, \mathrm{Sr}$, and $\mathrm{O}$ elements. The distribution of elements matches well with the shape of nanofibers, which indicate that the porous $\mathrm{SrTiO}_{3}$ nanofibers have been successfully prepared. Figures $2(\mathrm{e})-2(\mathrm{j})$ show the presence of $\mathrm{Ti}, \mathrm{Sr}, \mathrm{O}, \mathrm{Mo}$, and $\mathrm{S}$ elements on the surface of $\mathrm{SrTiO}_{3} @ \mathrm{MoS}_{2}$ hybrids, proving that the $\mathrm{MoS}_{2}$ nanosheets have been successfully adhere to the nanofibers.

The crystallographic structure of the prepared materials is shown in Figure 3, illustrated by the XRD profiles. The porous $\mathrm{SrTiO}_{3}$ nanofibers and pure $\mathrm{MoS}_{2}$ are precisely indexed to the JCPDS 35-0734 and JCPDS 37-1492, respectively. For 2D $\mathrm{SrTiO}_{3} @ \mathrm{MoS}_{2}$ composite nanofibers, the XRD peaks of $\mathrm{SrTiO}_{3}$ appeared at $23^{\circ}, 32^{\circ}, 40^{\circ}, 47^{\circ}, 52^{\circ}, 58^{\circ}, 68^{\circ}$, and $77^{\circ}$ can be assigned to the (100), (110), (111), (200), (210), (211), (220), and (310) planes, respectively. Nevertheless, the peaks of
$\mathrm{MoS}_{2}$ are very weak or deviated in the composite XRD patterns. The $\mathrm{V}-\mathrm{MoS}_{2}$ delaminated in the porous nanofibers may be due to the crystalline form changed. Interestingly, we can see some heteropeaks in the composite XRD patterns, and we discovered that the heteropeaks can fit well with the elemental sulfur. Thus, we inferred that not all the thioacetamide transferred to the $\mathrm{MoS}_{2}$, a small amount of thioacetamide transferred to elemental $S$ and adhered to the nanofibers, and causes some heteropeaks.

In order to illustrate the high photoelectric conversion capacity of the $\mathrm{SrTiO}_{3} @ \mathrm{MoS}_{2}$ composite nanofibers, the mechanism illustration is shown in Figure 4. Under simulate sunlight illumination, the porous $\mathrm{SrTiO}_{3}$ nanofibers produce photogenerated electrons, and the holes were left in the nanofibers. For the pure $\mathrm{SrTiO}_{3}$, the electrons and holes will recombine quickly and decrease the photoelectric performance immensely. However, after composing the V-MoS 2 , the conduction band position of $\mathrm{SrTiO}_{3}$ is much higher than $\mathrm{MoS}_{2}$. The photogenerated electrons will transfer to $\mathrm{MoS}_{2}$ and restrain the recombine of electrons and holes, which make the electrons more mobile and promote the photoelectric performance. Therefore, the $\mathrm{V}-\mathrm{MoS}_{2}$ structure can enlarge the surface area of materials and increase the active sites. In addition, the particular structure can retard the recombination of electrons and holes and then enhance the prolong of photoelectrons.

3.2. Optoelectronic Performance Test. Photocurrents and the electrochemical impedance spectroscopy (EIS) were used to explore the photoelectric conversion sensitivity and electronic transmission rate of the as-prepared samples. Figure 5(a) shows the I-T curves tested in the condition of the on-off cycle simulated sunlight for the pure $\mathrm{SrTiO}_{3}$ nanofibers and $\mathrm{SrTiO}_{3} @ \mathrm{MoS}_{2}$ heterostructure. The result of 


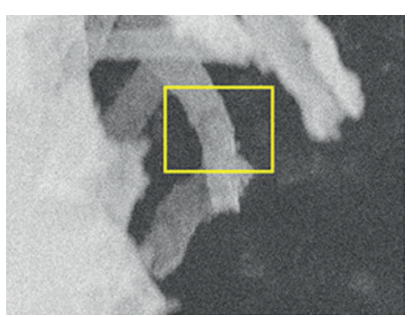

(a)

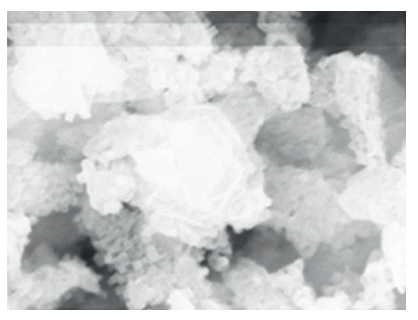

(e)

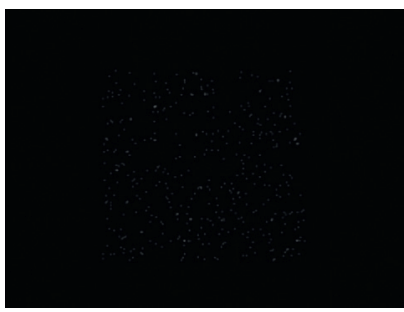

(i)

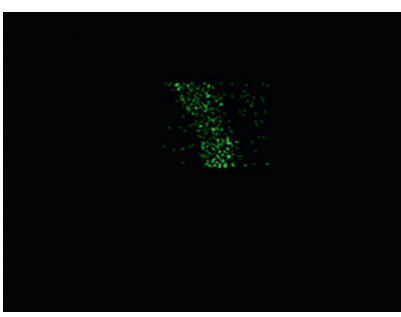

(b)

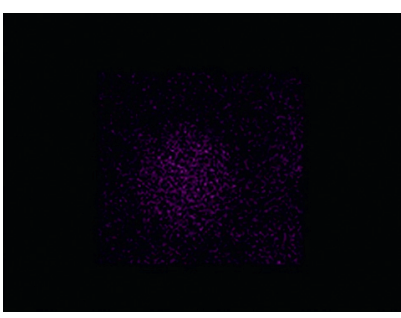

(f)

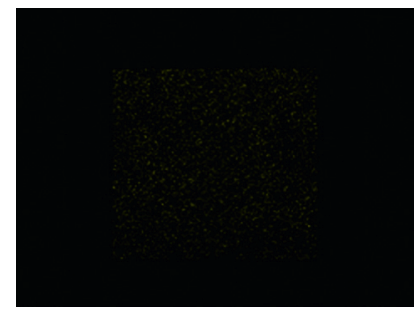

(j)

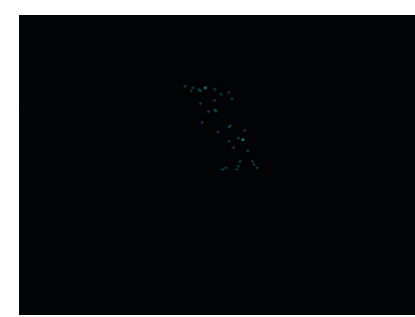

(c)

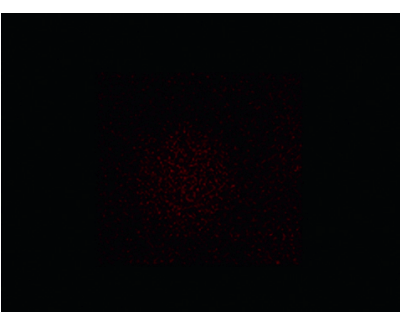

(g)

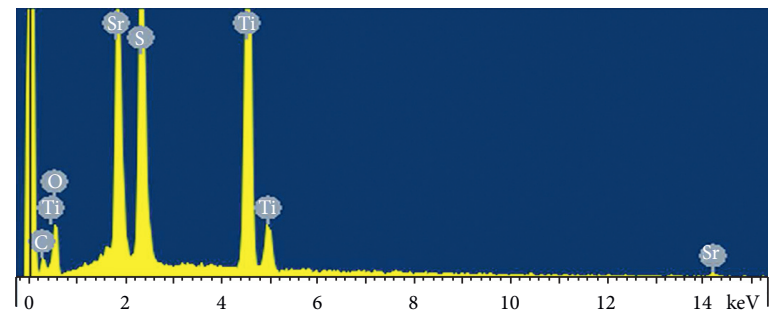

(k)

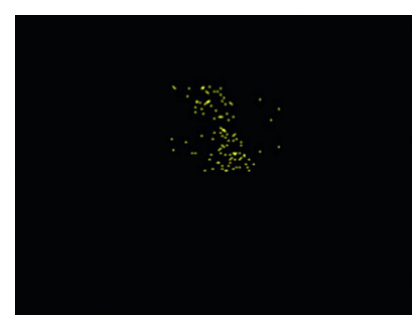

(d)

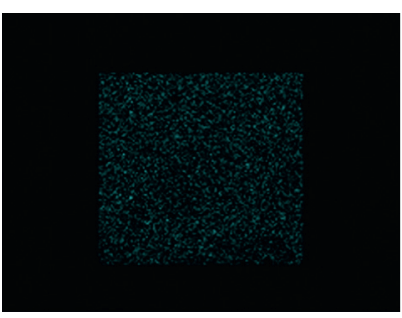

(h)

Figure 2: SEM image of (a) porous $\mathrm{SrTiO}_{3}$ nanofibers with (b-d) Ti/S/Sr elemental mapping and $\mathrm{SEM}$ image of (e) $\mathrm{SrTiO}_{3} @ M$ MoS composite $_{2}$ nanofibers with (f-j) $\mathrm{Mo} / \mathrm{S} / \mathrm{Ti} / \mathrm{S} / \mathrm{Sr} / \mathrm{O}$ elemental mapping and (k) EDX images of $\mathrm{SrTiO}_{3} @ \mathrm{MoS}_{2}$ composite nanofibers.

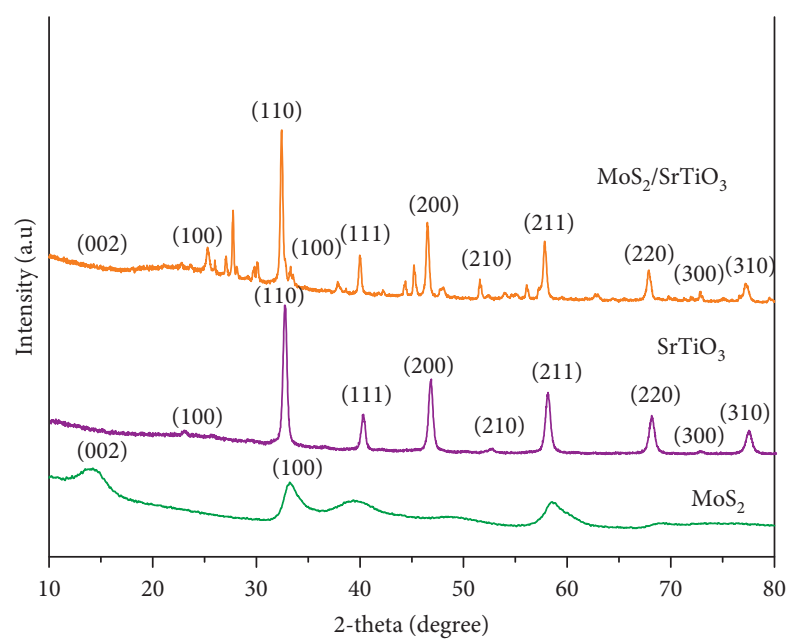

FIgURE 3: XRD patterns of pure $\mathrm{MoS}_{2}$ nanosheets, porous $\mathrm{SrTiO}_{3}$ nanofibers, and $\mathrm{SrTiO}_{3} @ \mathrm{MoS}_{2}$ composite nanofibers.

$\mathrm{SrTiO}_{3} @ \mathrm{MoS}_{2}$ indicates the photocurrent density is $21.4 \mu \mathrm{A}$, which is nearly twice higher than the pure $\mathrm{SrTiO}_{3}$ nanofibers $(10.1 \mu \mathrm{A})$. These results can prove that the adhering of $\mathrm{MoS}_{2}$ improves the conductivity of materials and promotes the electron transfer rate. In addition, the $\mathrm{V}-\mathrm{MoS}_{2}$ nanosheets can increase the surface area of nanofibers and improve the photosensitivity and the separation of photo-induced carriers. It is also worth noting that photocurrent curves show a regular rectangle shape, indicating the stability of composite materials. The EIS results in Figure 5(b) were applied to confirm the conductivity of the prepared composite. Moreover, the Rct of $\mathrm{SrTiO}_{3} @ \mathrm{MoS}_{2}$ is lower than that of porous $\mathrm{SrTiO}_{3}$ nanofibers, indicating that the $\mathrm{V}-\mathrm{MoS}_{2}$ on the nanofibers can accelerate the interfacial transfer and separation of charge carriers and then improve the optoelectronic performance. The use of $\mathrm{MoS}_{2}$ to improve the 


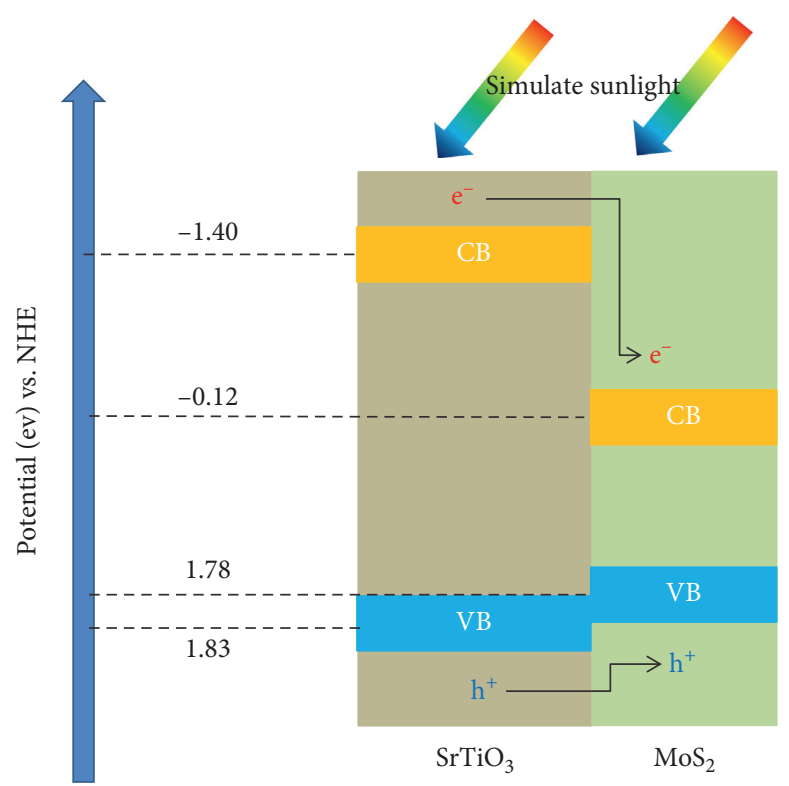

FIgURE 4: Schematic diagram of the photoelectric conversion mechanism of $\mathrm{SrTiO}_{3} @ \mathrm{MoS}_{2}$ composite nanofibers.

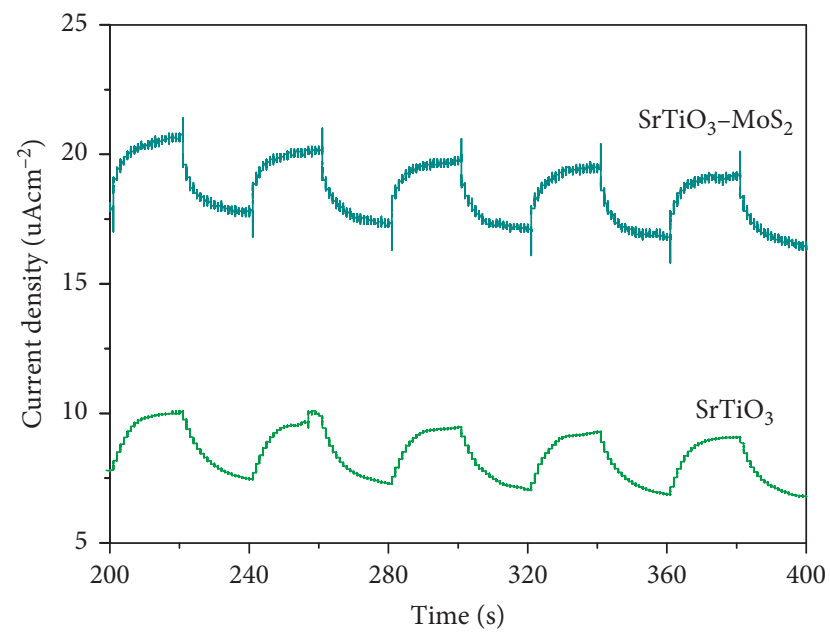

(a)

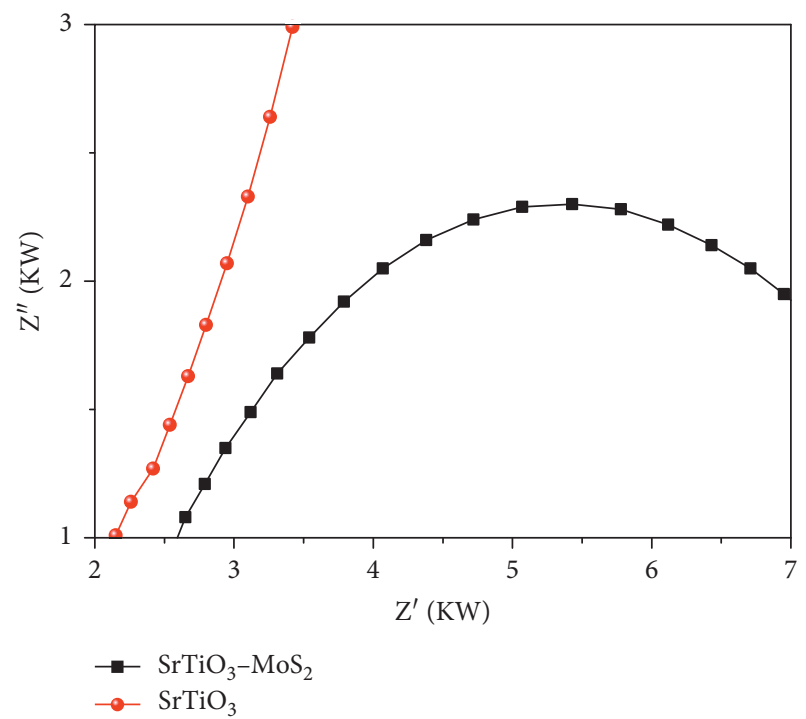

(b)

Figure 5: (a) The current density curves as a function of the time for $\mathrm{SrTiO}_{3}$ and $\mathrm{SrTiO}_{3} @ \mathrm{MoS}_{2}$ at $1.23 \mathrm{~V}$ in $1 \mathrm{M} \mathrm{KOH}$ electrolyte; (b) Nyquist plots of samples at $-0.24 \mathrm{~V}$ versus RHE measured from EIS in the frequency range from $100 \mathrm{kHz}$ to $0.1 \mathrm{~Hz}$.

performance of materials can be included in two reasons: first, the $\mathrm{MoS}_{2}$ improves the electronic orbital structure of the $\mathrm{SrTiO}_{3}$ and prolongs the life of photoelectrons. Then, constructing the structure of $\mathrm{V}-\mathrm{MoS}_{2}$ enlarges the surface area of materials, which means a larger contact area between electrodes and electrolytes.

\section{Conclusions}

In summary, new $\mathrm{SrTiO}_{3} @ \mathrm{MoS}_{2}$ composite materials were successfully synthesized. The structure and morphologies of the prepared composite were studied by SEM, TEM, EDX, XRD, I-T curves, and EIS analysis. The large surface area of the obtained $\mathrm{SrTiO}_{3}$ NFs can provide suitable sites for loading $\mathrm{MoS}_{2}$. Under simulate sunlight at a potential of $1.23 \mathrm{~V}$, the prepared composite materials exhibited a superior photoelectric performance of photocurrent density of $21.4 \mu \mathrm{A}$ and a resistance of $2.3 \Omega$. This study provides new clues for the preparation of $\mathrm{MoS}_{2}$-based composite materials and is widely used in the field of photodetector.

\section{Data Availability}

The experimental data used to support the findings of this study are included in the manuscript. The other data are available from the corresponding author upon request. 


\section{Conflicts of Interest}

The authors declare that there are no conflicts of interest.

\section{Acknowledgments}

The authors greatly appreciate the financial supports of National Natural Science Foundation of China (no. 21872119), Qinhuangdao Science and Technology Support Project (no. 201602A112), Science and Technology Support Project of Department of Health of Hebei Province (no. 20171246), and Project of Human Resources and Social Security Department of Hebei Province (no. A2017002075).

\section{References}

[1] S. Shi, Z. Sun, and Y. H. Hu, "Synthesis, stabilization and applications of 2-dimensional $1 \mathrm{~T}$ metallic $\mathrm{MoS}_{2}$," Journal of Materials Chemistry A, vol. 6, no. 47, pp. 23932-23977, 2018.

[2] J. Kou, Y. Liu, Y. Zhu, and J. Zhai, "Progress in piezotronics of transition-metal dichalcogenides," Journal of Physics D: Applied Physics, vol. 51, no. 49, p. 493002, 2018.

[3] Y. Fang, Y. Ge, C. Wang, and H. Zhang, "Mid-infrared photonics using 2D materials: status and challenges," Laser and Photonics Reviews, vol. 14, no. 1, Article ID 1900098, 2020.

[4] R. Hany, M. Cremona, and K. Strassel, "Recent advances with optical upconverters made from all-organic and hybrid materials," Science and Technology of Advanced Materials, vol. 20, no. 1, pp. 497-510, 2019.

[5] B. Rahmati, I. Hajzadeh, R. Karimzadeh, and S. M. Mohseni, "Facile, scalable and transfer free vertical-MoS2 nanostructures grown on $\mathrm{Au} / \mathrm{SiO} 2$ patterned electrode for photodetector application," Applied Surface Science, vol. 455, pp. 876-882, 2018.

[6] S. Ni, F. Guo, D. Wang et al., “Optimal Sr-Doped Free TiO $\mathrm{T}_{2} @$ $\mathrm{SrTiO}_{3}$ heterostructured nanowire arrays for high-efficiency self-powered photoelectrochemical UV photodetector applications," Crystals, vol. 9, no. 3, p. 134, 2018.

[7] X. Lu, P. Jiang, and X. Bao, "Phonon-enhanced photothermoelectric effect in $\mathrm{SrTiO}_{3}$ ultra-broadband photodetector," Nature Communications, vol. 10, no. 1, p. 138, 2019.

[8] W. Yang, Y. Zhang, Y. Zhang, W. Deng, and X. Fang, "Transparent Schottky photodiode based on AgNi NWs/ $\mathrm{SrTiO}_{3}$ contact with an ultrafast photoresponse to shortwavelength blue light and UV-shielding effect," Advanced Functional, Materials, vol. 29, no. 46, Article ID 1905923, 2019.

[9] X. Cui, Y. Wang, G. Jiang et al., "A photonic crystal-based $\mathrm{CdS}-\mathrm{Au}-\mathrm{WO}_{3}$ heterostructure for efficient visible-light photocatalytic hydrogen and oxygen evolution," RSC Advances, vol. 4, no. 30, pp. 15689-15694, 2014.

[10] S. Zhou, Y. Liu, J. Li et al., "Facile in situ synthesis of graphitic carbon nitride $\left(\mathrm{g}-\mathrm{C}_{3} \mathrm{~N}_{4}\right)-\mathrm{N}-\mathrm{TiO}_{2}$ heterojunction as an efficient photocatalyst for the selective photoreduction of $\mathrm{CO}_{2}$ to $\mathrm{CO}$," Applied Catalysis B: Environmental, vol. 159, pp. 20-29, 2014.

[11] X. Cui, Y. Wang, G. Jiang et al., "The encapsulation of CdS in carbon nanotubes for stable and efficient photocatalysis," Journal of Materials Chemistry, vol. 2, no. 48, pp. 2093920946, 2014.

[12] X. Cui, G. Jiang, M. Zhu et al., " $\mathrm{TiO}_{2} / \mathrm{CdS}$ composite hollow spheres with controlled synthesis of platinum on the internal wall for the efficient hydrogen evolution," International Journal of Hydrogen Energy, vol. 38, no. 22, pp. 9065-9073, 2013.
[13] J. Xiong, J. Li, J. Shi et al., "Metallic $1 \mathrm{~T}-\mathrm{MoS}_{2}$ nanosheets insitu entrenched on $\mathrm{N}, \mathrm{P}, \mathrm{S}$-codoped hierarchical carbon microflower as an efficient and robust electro-catalyst for hydrogen evolution," Applied Catalysis B: Environmental, vol. 243, pp. 614-620, 2019.

[14] J. Liu, L. Zhang, N. Li, Q. Tian, J. Zhou, and Y. Sun, "Synthesis of $\mathrm{MoS}_{2} / \mathrm{SrTiO}_{3}$ composite materials for enhanced photocatalytic activity under UV irradiation," Journal of Materials Chemistry A, vol. 3, no. 2, pp. 706-712, 2015.

[15] L. Wang, X. Liu, J. Luo et al., "Self-optimization of the active site of molybdenum disulfide by an irreversible phase transition during photocatalytic hydrogen evolution," Angewandte Chemie International Edition, vol. 56, no. 26, pp. 7610-7614, 2017.

[16] H. Zhou, Y. Liu, L. Zhang, H. Li, H. Liu, and W. Li, "Transition metal-doped amorphous molybdenum sulfide/graphene ternary cocatalysts for excellent photocatalytic hydrogen evolution: synergistic effect of transition metal and graphene," Journal of Colloid and Interface Science, vol. 533, no. 3, pp. 287-296, 2019.

[17] Y. Fu, X. Chen, X. Mou, Z. Ren, X. Li, and G. Han, “A dualcolor luminescent localized drug delivery system with ratiometric-monitored doxorubicin release functionalities," ACS Biomaterials Science \& Engineering, vol. 2, no. 4, pp. 652-661, 2016.

[18] H. Bai, Z. Liu, and D. D. Sun, "Facile fabrication of $\mathrm{TiO}_{2} /$ $\mathrm{SrTiO}_{3}$ composite nanofibers by electrospinning for high efficient $\mathrm{H}_{2}$ generation," Journal of the American Ceramic Society, vol. 96, no. 3, pp. 942-949, 2013.

[19] Z. Cao, C. Wang, and J. Chen, "Synthesis and photocatalytic property of $\mathrm{p}-\mathrm{n}$ junction $\mathrm{YMnO}_{3} / \mathrm{SrTiO}_{3}$ composites," $\mathrm{Ma}$ terials Research Express, vol. 5, no. 11, Article ID 115512, 2018.

[20] D. Hou, X. Hu, W. Ho, P. Hu, and Y. Huang, "Facile fabrication of porous Cr-doped SrTiO3 nanotubes by electrospinning and their enhanced visible-light-driven photocatalytic properties," Journal of Materials Chemistry A, vol. 3, no. 7, pp. 3935-3943, 2015.

[21] C. Grandclément, A. Piram, M.-E. Petit et al., "Biological removal and fate assessment of diclofenac using Bacillus subtilis and brevibacillus laterosporus strains and ecotoxicological effects of diclofenac and 4'-Hydroxy-diclofenac," Journal of Chemistry, vol. 2020, Article ID 9789420, 12 pages, 2020.

[22] J. Zhao, P. Zhang, J. Fan, J. Hu, and G. Shao, "Constructing 2D layered $\mathrm{MoS}_{2}$ nanosheets-modified Z-scheme $\mathrm{TiO}_{2} / \mathrm{WO}_{3}$ nanofibers ternary nanojunction with enhanced photocatalytic activity," Applied Surface Science, vol. 430, pp. 466474, 2018

[23] H. Hou, F. Gao, L. Wang et al., "Superior thoroughly mesoporous ternary hybrid photocatalysts of $\mathrm{TiO}_{2} / \mathrm{WO}_{3} / \mathrm{g}-\mathrm{C}_{3} \mathrm{~N}_{4}$ nanofibers for visible-light-driven hydrogen evolution," Journal of Materials Chemistry A, vol. 4, no. 17, pp. 6276-6281, 2016.

[24] L. Zhang, J. Yin, K. Wei et al., "Fabrication of hierarchical $\mathrm{SrTiO}_{3} @ \mathrm{MoS}_{2}$ heterostructure nanofibers as efficient and lowcost electrocatalysts for hydrogen-evolution reactions," Nanotechnology, vol. 31, no. 20, Article ID 205604, 2020.

[25] X. Yue, S. Yi, R. Wang, Z. Zhang, and S. Qiu, "Well-controlled SrTiO3@Mo2C core-shell nanofiber photocatalyst: boosted photo-generated charge carriers transportation and enhanced catalytic performance for water reduction," Nano Energy, vol. 47, pp. 463-473, 2018.

[26] Y. Li, N. Luo, Z. Tian et al., " $\mathrm{H}_{2} \mathrm{O}_{2}$-free photo-Fenton degradation of organic pollutants on thermally exfoliated 
g- $\mathrm{C}_{3} \mathrm{~N}_{4}$," Colloids and Surfaces A: Physicochemical and Engineering Aspects, vol. 586, Article ID 124190, 2020.

[27] M. Yang, R. Liu, H. Chen, H. Li, and P. Guo, "Synthesis of selfassembled nickel cobaltite microspheres and their electrocapacitive behavior in aqueous electrolytes," Colloids and Surfaces A: Physicochemical and Engineering Aspects, vol. 587, Article ID 124329, 2020.

[28] J. Sun, M. Yang, Y. Gong, H. Li, and P. Guo, "Synthesis of $\mathrm{Pd}_{3} \mathrm{~Pb}$ colloidal nanocrystal assembly and their electrocatalytic activity toward ethanol oxidation," Colloids and Surfaces A: Physicochemical and Engineering Aspects, vol. 586, Article ID 124224, 2020.

[29] X. Yang, Z. Tian, Y. Chen, H. Huang, and J. Hu, "One-pot calcination preparation of graphene/g- $\mathrm{C}_{3} \mathrm{~N}_{4}$-Co photocatalysts with enhanced visible light photocatalytic activity," International Journal of Hydrogen Energy, vol. 45, no. 23, pp. 12889-12902, 2020.

[30] L. Ge, M. Zhang, R. Wang et al., "Fabrication of CS/GA/RGO/ $\mathrm{Pd}$ composite hydrogels for highly efficient catalytic reduction of organic pollutants," RSC Advances, vol. 10, no. 26, pp. 15091-15097, 2020.

[31] J. Yin, F. Zhan, T. Jiao et al., "Facile preparation of self-assembled MXene@Au@CdS nanocomposite with enhanced photocatalytic hydrogen production activity," Science China Materials, vol. 63, 2020.

[32] J. Yin, Q. Liu, J. Zhou et al., "Self-assembled functional components-doped conductive polypyrrole composite hydrogels with enhanced electrochemical performances," RSC Advances, vol. 10, no. 18, pp. 10546-10551, 2020.

[33] R. Wang, X. Yan, B. Ge et al., "Facile preparation of selfassembled black phosphorus-dye composite films for chemical gas sensors and surface-enhanced Raman scattering performances," ACS Sustainable Chemistry \& Engineering, vol. 8, no. 11, pp. 4521-4536, 2020.

[34] K. Ma, R. Wang, Y. Rao, W. Zhao, S. Liu, and T. Jiao, "Langmuir-Blodgett films of two chiral perylene bisimidebased molecules: aggregation and supramolecular chirality," Colloids and Surfaces A: Physicochemical and Engineering Aspects, vol. 591, Article ID 124563, 2020.

[35] Y. Feng, J. Yin, S. Liu, Y. Wang, B. Li, and T. Jiao, "Facile synthesis of $\mathrm{Ag} / \mathrm{Pd}$ nanoparticle-loaded poly(ethylene imine) composite hydrogels with highly efficient catalytic reduction of 4-nitrophenol," ACS Omega, vol. 5, no. 7, pp. 3725-3733, 2020.

[36] Y. He, R. Wang, C. Sun et al., "Facile synthesis of self-assembled NiFe layered double hydroxide-based azobenzene composite films with photoisomerization and chemical gas sensor performances," ACS Omega, vol. 5, no. 7, pp. 36893698, 2020.

[37] J. Song, C. Yuan, T. Jiao et al., "Multifunctional antimicrobial biometallohydrogels based on amino acid coordinated selfassembly," Small, vol. 16, no. 8, Article ID 1907309, 2020.

[38] C. Cai, R. Wang, S. Liu et al., "Synthesis of self-assembled phytic acid-mxene nanocomposites via a facile hydrothermal approach with elevated dye adsorption capacities," Colloids and Surfaces A: Physicochemical and Engineering Aspects, vol. 589, Article ID 124468, 2020.

[39] Y. Xu, R. Wang, Y. Zheng et al., "Facile preparation of selfassembled Ni/Co phosphates composite spheres with highly efficient her electrocatalytic performances," Applied Surface Science, vol. 509, Article ID 145383, 2020.

[40] R. Geng, J. Yin, J. Zhou et al., "In situ construction of Ag/ $\mathrm{TiO}_{2} / \mathrm{g}-\mathrm{C}_{3} \mathrm{~N}_{4}$ heterojunction nanocomposite based on hierarchical co-assembly with sustainable hydrogen evolution," Nanomaterials, vol. 10, no. 1, p. 1, 2020.

[41] F. Zhan, J. Yin, J. Zhou et al., "Facile preparation and highly efficient catalytic performances of Pd-Cu bimetallic catalyst synthesized via Seed-mediated method," Nanomaterials, vol. 10, no. 1, p. 6, 2020.

[42] Y. Feng, R. Wang, J. Yin et al., "Facile synthesis of $\mathrm{Cu}_{2} \mathrm{O}$ nanoparticle-loaded carbon nanotubes composite catalysts for reduction of 4-Nitrophenol," Current Nanoscience, vol. 16, no. 4, pp. 611-618, 2020.

[43] J. Zhao, J. Yin, J. Zhong et al., "Facile preparation of a selfassembled Artemia cyst shell- $\mathrm{TiO}_{2}-\mathrm{MoS}_{2}$ porous composite structure with highly efficient catalytic reduction of nitro compounds for wastewater treatment," Nanotechnology, vol. 31, no. 8, p. 85603, 2020.

[44] Y. Meng, J. Yin, T. Jiao et al., "Self-assembled copper/cobaltcontaining polypyrrole hydrogels for highly efficient ORR electrocatalysts," Journal of Molecular Liquids, vol. 298, Article ID 112010, 2020

[45] J. Yin, F. Zhan, T. Jiao et al., "Highly efficient catalytic performances of nitro compounds via hierarchical PdNPs-loaded MXene/polymer nanocomposites synthesized through electrospinning strategy for wastewater treatment," Chinese Chemical Letters, vol. 31, no. 4, pp. 992-995, 2020.

[46] K. Ma, R. Wang, T. Jiao et al., "Preparation and aggregate state regulation of co-assembly graphene oxide-porphyrin composite Langmuir films via surface-modified graphene oxide sheets," Colloids and Surfaces A: Physicochemical and Engineering Aspects, vol. 584, Article ID 124023, 2020.

[47] X. Xu, Y. Liu, W. Fu et al., "Poly(N-isopropylacrylamide)based thermoresponsive composite hydrogels for biomedical applications," Polymers, vol. 12, no. 3, p. 580, 2020. 